\title{
La teoría cuántica de átomos en moléculas y su rol en la reducción de la química a la física*
}

The Quantum Theory of Atoms in Molecules and Its Role in the Reduction of Chemistry to Physics

Jesús Alberto Jaimes Arriaga ${ }^{\dagger}$

Sebastian Fortin ${ }^{\ddagger}$

\begin{abstract}
Resumen
El problema de la reducción entre química y física ha sido abordado principalmente a través de la mecánica cuántica estándar y la química clásica estructural. En este trabajo la estudiaremos desde la perspectiva de una teoría alternativa a la cuántica estándar, la mecánica Bohmiana y la teoría cuántica de átomos en moléculas (TCAEM) propuesta por Bader. El propósito de este artículo se centra en develar el papel que desempeña la TCAEM en las relaciones interteóricas entre química y física. Argumentaremos que si bien la TCAEM supera dos grandes obstáculos al brindar una definición rigurosa de enlace químico y átomos dentro de una molécula, la teoría hace uso de nociones clásicas incompatibles con los principios de la mecánica cuántica tradicional y por lo tanto no logra la reducción. Por otro lado mostraremos que la TCAEM guarda mayor similitud con la mecánica Bohmiana y que los elementos básicos de ambas teorías resultan afines.
\end{abstract}

Palabras clave: mecánica Bohmiana - teoría cuántica de átomos en moléculas - química - física - reducción

\begin{abstract}
The problem of reduction between chemistry and physics has been addressed mainly through standard quantum mechanics and classical structural chemistry. In this work, we will study it from the perspective of an alternative theory to standard quantum, Bohmian mechanics and Quantum Theory of Atoms in Molecules (QTAIM) proposed by Bader. The purpose of this article is to unveil the role of QTAIM in the inter-theoretical relations between chemistry and physics. We argue that although QTAIM solve two obstacles by providing a rigorous definition of chemical bond and atoms in a molecule, the theory uses classical concepts unacceptable in the quantum mechanical context. Therefore, it fails to achieve reduction. On the other hand, we will show that the TCAEM is more similar to the Bohmian mechanics and the basic elements of both theories are related.
\end{abstract}

Keywords: Bohmian mechanics - quantum theory of atoms in molecules - chemistry - physics - reduction

\footnotetext{
* Recibido: 27 de Mayo de 2018. Aceptado con revisiones: 6 de Septiembre de 2018.

† Universidad de Buenos Aires/CONICET, Argentina. Para contactar al autor, por favor, escribir a: 304156359@comunidad.unam.mx.

* CONICET/FCEN, Universidad de Buenos Aires, Argentina. Para contactar al autor, por favor, escribir a: sfortin@conicet.gov.ar. Metatheoria 9(2)(2019): 33-43. ISSN 1853-2322. eISSN 1853-2330.

(c) Editorial de la Universidad Nacional de Tres de Febrero. Publicado en la República Argentina.
} 


\section{Introducción}

En los últimos años la filosofía de la química ha experimentado un importante crecimiento en cuanto a su campo de estudio. Aún con ello, la relación entre química y física sigue siendo de particular relevancia dentro de esta disciplina, ya que afecta la independencia de la química como disciplina científica. Dentro de esta temática, el principal problema surge cuando la mecánica cuántica trata de dar cuenta de la estructura molecular. Y es en este aspecto que en los últimos años ha logrado un creciente interés la teoría cuántica de átomos en moléculas (TCAEM), desarrollada por el químico cuántico canadiense Richard Bader (1994), tanto en la comunidad científica (Matta, Massa \& Keith 2011) como en la filosófica (Matta 2013), ya que ofrece una fundamentación teórica rigurosa, basada en la mecánica cuántica, para la noción química de estructura molecular.

El problema de la reducción entre química y física ha sido abordado en su mayoría a través de la mecánica cuántica, dejando de lado otros formalismos como el de la mecánica Bohmiana. Bajo esta consideración, Bader propone la TCAEM como una extensión de la mecánica cuántica para el estudio de los sistemas químicos, y no como una alternativa para obtener información de la función de onda (Bader 1998). Dentro de esta teoría, la estructura molecular se define en términos de la densidad electrónica y se hace uso de los teoremas de la mecánica cuántica para dar cuenta de la noción de enlace químico. De esta manera la teoría propone un marco reduccionista que sienta las bases de una teoría física unificadora de la estructura molecular. Sin embargo, mucho del lenguaje empleado por la TCAEM puede considerarse diametralmente opuesto a los presupuestos que emplea la mecánica cuántica, y por otro lado la acercan al enfoque clásico determinista que caracteriza a la mecánica Bohmiana. Por ello resulta interesante analizar qué relación guarda la TCAEM con respecto a estas dos teorías, la mecánica cuántica y la mecánica Bohmiana.

El propósito de este artículo se centra en develar el papel que desempeña la TCAEM en las relaciones interteóricas entre química y física, en el caso particular de la reducción de la química molecular a mecánica cuántica. Argumentaremos en favor de la idea de que la TCAEM guarda mayor similitud con la mecánica Bohmiana, mientras se aleja de los supuestos planteados por la mecánica cuántica. Para ello este trabajo ha sido estructurado de la siguiente manera, en la Sección 2, "¿Sobre qué trata la TCAEM?", se hace una breve presentación de los principales rasgos de la teoría. En la Sección 3, "Mecánica cuántica ortodoxa", se introducen los problemas fundacionales más importantes de la mecánica cuántica. En la Sección 4, "La mecánica cuántica Bohmiana”, está destinada a explicar las cualidades que caracterizan a la mecánica Bohmiana. Dentro de la Sección 5, “Dónde queda la TCAEM?", se discuten las semejanzas y diferencias que guardan la TCAEM con las otras dos teorías. Y finalmente, en las conclusiones se destacarán los resultados a los que se llegaron y los aspectos filosóficos que aún quedan por abordar.

\section{2. ¿Sobre qué trata la TCAEM?}

Dentro del contexto de la TCAEM, Bader señala que la manifestación de la materia en el espacio real está representada por la distribución espacial de la densidad electrónica asociada a los sistemas que dan forma a la materia, es decir, los átomos y las moléculas. Desde su perspectiva esta densidad electrónica contiene toda la información física de la materia y su topología incluye los conceptos de átomo, enlace, estructura y estabilidad estructural. Así, una teoría basada en la densidad electrónica puede establecer un enlace entre el lenguaje de la química y el de la física (Bader 2011).

La primera definición de densidad electrónica fue formulada por Schrödinger (1926b, 1926c) como una distribución continua de electricidad en el espacio y, además, él enfatiza que la función de onda, $\psi$ no debe ser usada directamente para interpretar el fenómeno físico, debido a que es una función en el espacio configuracional y no en el espacio real. En su lugar, aboga por que el uso de la 
función de onda, $\psi$ sea exclusivamente para el propósito de obtener la cantidad $\psi^{*} \psi$, ya que "la carga del electrón no está concentrada en un punto, sino que esta esparcida a través del espacio total, proporcional a la cantidad $\psi^{*} \psi$ " (Schrödinger 1926c, p. 1066). Así, Schrödinger obtiene la densidad electrónica $\rho(r)$ integrando sobre las coordenadas de todos excepto uno de los electrones, al cual mantiene fijo en el punto $r$, donde se quiere conocer la densidad electrónica, y entonces calcula la suma de estas integrales para el resto de los electrones. Adicionalmente se debe hacer una corrección, considerar el espín electrónico (Bader 2010). De esta manera se obtiene,

$$
\rho(r)=N \int \psi^{*} \psi d \tau
$$

En la TCAEM (Bader 1991, 1994) la estructura molecular es caracterizada por la topología de la distribución electrónica $\rho(r)$, donde la morfología dominante define un átomo en una molécula (Bader 2005). Esta morfología está dada por la cantidad y tipo de características topológicas de la distribución. Tales características están asociadas con puntos críticos, en los cuales la primera derivada de $\rho(r)$ se anula. Así mismo, la segunda derivada de $\rho(r)$ distingue las diferentes características topológicas, particularmente, máximos, mínimos y sillas. En este contexto, las posiciones de los núcleos corresponden a máximos de la distribución, y esta característica topológica conduce a la partición natural del espacio molecular en regiones mononucleares, las cuales, en la TCAEM, son identificadas con los átomos de la molécula. La superficie que limita un átomo en una molécula corresponde a una superficie de flujo cero en el campo vectorial gradiente, ya que se interpreta que la densidad electrónica asociada con tal región (un átomo) permanece sin cambio a través del tiempo. De acuerdo con Bader, la asociación de estas características topológicas con los elementos de la estructura molecular sienta las bases para una rigurosa teoría de estructura química.

Conforme a Bader, existen cuatro diferentes tipos de puntos críticos estables, los cuales son caracterizados por su rango, ù y signatura, ó. El rango es el número de curvas diferentes de cero, esto es las dimensiones del espacio y la signatura la suma algebraica de los signos de estas curvas (Bader 1994). En este sentido, tenemos un punto crítico asociado a posiciones nucleares $(3,-3)$, que es un máximo local en la distribución electrónica, así mismo tenemos un mínimo local, un punto crítico $(3,+3)$ correspondiente a una "jaula" de átomos. Finalmente tenemos dos tipos de silla, los puntos críticos (3, -1) y $(3,+1)$; el primero encontrado entre dos átomos que están enlazados y el segundo dentro de un anillo de átomos también enlazados. Con la ayuda de estas características de la topología de la distribución electrónica, es posible construir la estructura molecular de cualquier molécula y, de esta manera, como el autor lo indica, el concepto de estructura molecular es recuperado por la física a través de la mecánica cuántica. En este contexto, la TCAEM ofrece un esquema reduccionista en el cual el autor aboga por el uso de la noción de vía de enlace en lugar de la de enlace químico, debido a que el concepto de enlace químico es insuficiente para dar cuenta de la totalidad de modelos de enlace empleados en la química. Igualmente, él señala que el concepto de vía de enlace es un indicador universal de que dos átomos están enlazados, y así cumple con la visión física unificada para todas las interacciones de enlace empleadas por los químicos (Bader 2009, 2011). En palabras de su autor,

El enfoque reduccionista proporcionado por la TCAEM ofrece una solución clara para la miríada de visiones personales y modelos de enlace. Como ha sido ampliamente demostrado por apelar a la física, la presencia de una vía de enlace conectando un par de átomos cumple las condiciones suficientes y necesarias de que los átomos están enlazados el uno con el otro. Esta definición, la cual necesariamente aplica a las densidades mecánico-cuánticas, trasciende todos los esquemas de enlace y categorías, y provee una compresión física unificada de las interacciones atómicas. Uno asume que tal unificación sea el objetivo principal de cualquier teoría física. (Bader 2011, p. 20)

Además, la noción de vía de enlace está enraizada en una cantidad mensurable, a saber, la densidad electrónica y es posible definir un operador de vía de enlace como un observable de Dirac, y así obtener los valores esperados correspondientes a esta propiedad. En este caso, se puede ver que cuando dos 
átomos interactúan el uno con el otro, ellos están enlazados por medio de una acumulación de densidad electrónica entre ellos (Bader 2009, 2011), un hecho ya observado en los primeros desarrollos de la química cuántica (Heitler \& London 1927). Además es posible asignar una cierta densidad electrónica a cada átomo en la molécula, la cual determina la contribución de dicho átomo a las propiedades del sistema entero, la molécula (Bader 2007).

Sobre estas bases, Bader plantea la posibilidad de usar los teoremas de la mecánica cuántica en la interpretación del enlace químico, una propuesta que había sido introducida previamente por Slater en 1933. Adicionalmente, la hipótesis usada por la TCAEM es que una molécula es una colección de átomos, y cada átomo contribuye a las propiedades de la molécula. De este modo, la TCAEM provee un fundamento físico para el concepto de grupo funcional.

\section{Mecánica cuántica ortodoxa}

La visión estándar de la mecánica cuántica está basada en la llamada interpretación de Copenhague. Este marco conceptual fue desarrollado principalmente por Niels Bohr junto con Werner Heisenberg, Max Born y otros físicos, en un intento por tratar de comprender el mundo atómico a través de la naciente teoría cuántica. Sin embargo, desde sus inicios, esta visión fue en contra de los fundamentos de la física clásica, ya que proponía principios contra intuitivos, tanto que personajes de la talla de Einstein o del mismísimo Schrödinger mantuvieron una actitud abiertamente en contra de esta interpretación, arguyendo que la teoría cuántica aún estaba incompleta y que una mejor descripción del mundo cuántico era posible (Bricmont 2016).

En 1926 Erwin Schrödinger publica una serie de artículos en los que desarrolla los principios de una nueva teoría que pretendía dar cuenta del comportamiento de las partículas que constituían la materia. Ciertamente, un año antes, Heisenberg ya había propuesto una teoría basada en matrices y propiedades que no conmutaban con el mismo propósito, no obstante más adelante se demostró que tanto esta teoría como la de Schrödinger eran equivalentes. La ecuación propuesta por Schrödinger puede ser escrita de la siguiente manera,

$$
i \hbar \frac{\partial}{\partial t} \Psi(r, t)=\left[-\frac{\hbar^{2}}{2 m} \bar{\nabla}^{2}+V(r, t)\right] \Psi(r, t)
$$

Donde $\hbar$ es la constante de Planck dividida por 2ð, $m$ es la masa de la partícula, $\nabla^{2}$ es el operador diferencial laplaciano, $V$ es la energía potencial del sistema y $\Psi$ es la función de onda que representa un estado del sistema cuántico. En el formalismo que se emplea actualmente, los estados se representan por vectores del espacio de Hilbert $H$, un espacio vectorial, en el cual los componentes de los vectores pueden ser complejos y cuyo producto vectorial da como resultado números reales con significado físico. En este contexto cada estado del sistema tiene asociado un vector $|\phi\rangle$ (ket, en notación de Dirac) perteneciente a un espacio de Hilbert, $|\phi\rangle \in H$. Así mismo, las propiedades medibles del sistema cuántico, es decir, los observables, están representados por operadores lineales que actúan sobre el espacio de Hilbert (Ballentine 1998). Para un observable determinado $O$, se tiene un operador $\hat{O}$, el cual tiene asociado autovectores $\left|o_{i}\right\rangle$ y autovalores $o_{i}$, de tal manera que cuando se aplica dicho operador a un autovector, se obtiene el mismo autovector multiplicado por el autovalor correspondiente, esto es $\hat{O}\left|o_{i}\right\rangle=o_{i}\left|o_{i}\right\rangle$.

De acuerdo a la mecánica cuántica, los autovalores $o_{1}, o_{2}, o_{3}, \ldots, o_{i}$ asociados a cada operador $\hat{O}$, son los valores posibles para el observable $O$, obtenidos cuando se lleva a cabo una medición de la propiedad en cuestión. A partir de estos operadores se pueden calcular magnitudes físicas de la siguiente manera: el valor medio de la propiedad $O$, asociada al operador $\hat{O}$, en el estado cuántico $|\phi\rangle$, se calcula como $\langle\hat{O}\rangle=\langle\phi|\hat{O}| \phi\rangle$.

Dentro de este formalismo matemático, los autovectores asociados a un operador $\hat{O}$, forman una base en el espacio de Hilbert $H$, esto significa que cualquier estado del sistema cuántico $|\phi\rangle$ puede ser escrito en términos de combinaciones lineales de estos autovectores. Pongamos un ejemplo, 
consideremos los estados $\left|a_{1}\right\rangle,\left|a_{2}\right\rangle,\left|a_{3}\right\rangle, \ldots,\left|a_{N}\right\rangle$ una base en el espacio de Hilbert $H$, tal que, podemos escribir el estado $|\phi\rangle$ como una combinación lineal de éstos,

$$
|\phi\rangle=\sum_{i=1}^{N} \mathrm{a}_{i}\left|a_{i}\right\rangle
$$

donde los coeficientes $a_{1}, a_{2}, a_{3}, \ldots, a_{N}$ son números complejos. Además, tenemos que, existe un número infinito de bases en el espacio de Hilbert, de tal forma que el mismo estado $|\phi\rangle$ se puede reescribir en términos de otra base, por ejemplo $\left|b_{1}\right\rangle,\left|b_{2}\right\rangle,\left|b_{3}\right\rangle, \ldots,\left|b_{N}\right\rangle$, así tendremos,

$$
|\phi\rangle=\sum_{i=1}^{N} \dot{a}_{i}\left|a_{i}\right\rangle=\sum_{i=1}^{N} \hat{\mathrm{a}}_{i}\left|b_{i}\right\rangle
$$

donde nuevamente los coeficientes $\hat{\mathrm{a}}_{1}, \hat{\mathrm{a}}_{2}, \hat{\mathrm{a}}_{3}, \ldots, \hat{\mathrm{a}}_{N}$ son números complejos que guardan una relación con los primeros, dependiente de qué vectores se emplearon en cada base.

A partir de esta característica matemática se sigue uno de los problemáticos principios de la mecánica cuántica, a saber, el principio de superposición, el cual dice que cualquier combinación lineal de estados posibles para un sistema, también es un estado válido para el sistema. Para analizarlo consideremos la propiedad de espín del electrón, dicha propiedad puede adquirir dos estados: espín arriba $|\uparrow\rangle$ o espín abajo $|\downarrow\rangle$. Pero además, la combinación lineal de éstos, o sea $|\phi\rangle=C_{\uparrow}|\uparrow\rangle+C_{\downarrow}|\downarrow\rangle$, también es un estado posible de esta propiedad, producto de la superposición de los estados espín arriba $|\uparrow\rangle$ y espín abajo $|\downarrow\rangle$. Nótese que, este nuevo estado de nuestro sistema no es ni espín arriba ni espín abajo, sino una combinación de ambos, lo cual resulta desconcertante desde la física clásica ya que en ella una propiedad tiene un estado definido y nunca una superposición de dos o más estados como en la cuántica.

Otra característica muy peculiar de la mecánica cuántica la encontramos en el principio de incertidumbre o indeterminación de Heisenberg, el cual se formula matemáticamente de la siguiente manera (Landau \& Lifshitz 1972),

$$
\Delta x \Delta p \geq \frac{\hbar}{2}
$$

y del cual se sigue, que si nosotros conocemos con exactitud la posición de una partícula, por ejemplo un electrón, no podremos obtener información precisa acerca de su momento (o velocidad, dado que $p=m v$ ) y viceversa. Este importante principio es consecuencia del formalismo matemático, en el cual las propiedades medibles de un sistema cuántico, los observables, se encuentran representadas por operadores incompatibles, es decir, cuyo conmutador es diferente de cero, $[\hat{A}, \hat{B}]=\hat{A} \hat{B}-\hat{B} \hat{A} \neq 0$. Para revisarlo con más detalle consideremos un sistema cuántico con la propiedad $A$, representada por su operador $\hat{A}$ y con sus autoestados $\left|a_{i}\right\rangle$, y con la propiedad $B$, representada por su operador $\hat{B}$ y con sus autoestados $\left|b_{i}\right\rangle$. En este caso, los autoestados del operador $\hat{A}$ son diferentes de los del operador $\hat{B}$, pero de acuerdo al formalismo del espacio de Hilbert revisado anteriormente, un determinado autoestado de $\hat{A}$ puede ser representado por una combinación lineal de autoestados de $\hat{B}$. Así, tenemos que

$$
\left|a_{i}\right\rangle=\sum_{i=1}^{N} \hat{\mathrm{a}}_{i j}\left|b_{j}\right\rangle
$$

donde el coeficiente $\hat{a}_{i j}$ no puede tomar los valores de cero y uno, esto significa que las bases $\left\{\left|a_{i}\right\rangle\right\}$ y $\left\{\left|b_{j}\right\rangle\right\}$ no coinciden en el espacio de Hilbert al que pertenecen, de tal manera que, cuando se encuentra bien definida la propiedad $A$ con el valor $a_{k}$, también está bien definido su autoestado $\left|a_{k}\right\rangle$, el cual, de acuerdo a la sumatoria anterior, puede ser expresado como una superposición de autoestados pertenecientes a la propiedad $B$, lo que significa que la propiedad $B$ se encuentra indefinida. En tal caso se dice que la mecánica cuántica es contextual, esto es, que únicamente admite la determinación simultánea de propiedades que comparten la misma base en un espacio de Hilbert (Bub 1997). 
Hasta este punto hemos hablado de los sistemas cuánticos como "objetos individuales", no obstante, considerando ciertos aspectos de la teoría, resulta dudoso considerarlos de esta manera. Tradicionalmente un objeto individual es un objeto que puede ser rastreado sin ambigüedad en el espacio y el tiempo, es independiente y diferente de otros, es el mismo a través del tiempo. Considerando lo anteriormente expuesto, se puede concluir que los sistemas cuánticos, en particular los electrones, protones y neutrones, no pueden ser considerados individuos en el sentido tradicional de la palabra. Esto se debe a varias razones, por un lado algunas de sus propiedades como la posición no se encuentran siempre definidas gracias a la contextualidad de la teoría, y por otro lado el requerimiento de simetrización (bosones) o antisimetrización (fermiones) de la función de onda obliga a interpretar a las entidades cuánticas como no- individuos (French \& Krause 2006). Esta pérdida de individualidad no es meramente una limitación epistémica, sino una ontológica, de implicaciones más profundas. Finalmente, en cuanto dos sistemas cuánticos independientes, por ejemplo electrón y protón, comienzan a interactuar, pierden su individualidad, ya que dan origen a un nuevo sistema, uno compuesto, como es el caso de la molécula de hidrógeno, el cual es tratado de manera holista y nolocal (Ballentine 1998).

\section{La mecánica cuántica Bohmiana}

Una teoría alternativa a la mecánica cuántica fue desarrollada por David Bohm a principios de los años cincuenta (Bohm 1952a, b). Dicha visión se basa en el empleo de variables ocultas para dar una descripción completa del fenómeno cuántico, y consigue una reconstrucción de la mecánica cuántica más acorde a los principios de la física clásica, ya que se pueden obtener las trayectorias de las partículas estudiadas.

Antes de comenzar con los principios de la mecánica Bohmiana, es conveniente recordar brevemente la formulación de Hamilton-Jacobi de la mecánica clásica, en la cual el movimiento de los cuerpos se obtiene a partir de la ecuación,

$$
\frac{\partial S}{\partial t}+\frac{(\bar{\nabla} S)^{2}}{2 m}+V=0
$$

donde $S$ es la acción y $V$ el potencial. A partir de este formalismo se puede calcular el momentum de un cuerpo de la siguiente manera (Goldstein et al. 2002),

$$
\bar{p}=\bar{\nabla} S
$$

En la mecánica Bohmiana, el movimiento de las partículas está dado en el espacio real de tres dimensiones y obedece la siguiente ecuación,

$$
\frac{d Q_{i}}{d t}=\frac{\hbar}{m_{i}} \operatorname{Im} \frac{\nabla_{i} \Psi\left(Q_{1}(t), \ldots, Q_{N}(t), t\right)}{\Psi\left(Q_{1}(t), \ldots, Q_{N}(t), t\right)}
$$

donde $I m$ representa la parte imaginaria de la ecuación, $Q_{i}(t) \in \mathbb{R}^{3}$ y $m_{i}$ representan la posición y masa de la i-ésima partícula, y $\Psi$ es la función de onda de Schrödinger que satisface la ecuación (2) (Tumulka 2004). Esta ecuación de movimiento es el resultado de la introducción de una nueva fuerza que resulta un poco misteriosa ya que depende de todas las partículas del universo. Para entender el origen de esta nueva fuerza partamos de la ecuación de Schrödinger (ecuación (2)), en la cual la función de onda $\varnothing$ es una función compleja, por lo que se puede escribir de la siguiente manera (Holland 1993),

$$
\varnothing=R e^{\frac{i S}{\hbar}}
$$

donde $R$ es el módulo de la función de onda, tal que $R^{2}=P$ es la densidad de probabilidad, y $S$ su fase. Cuando introducimos la ecuación (8) en la (2), y realizamos el cambio de variable $R \rightarrow P$, obtenemos las siguientes ecuaciones, 


$$
\begin{gathered}
\frac{\partial P}{\partial t}+\bar{\nabla} \cdot\left(P \frac{\nabla S}{m}\right)=0 \\
\frac{\partial S}{\partial t}+\frac{(\bar{\nabla} S)^{2}}{2 m}+V-\frac{\hbar^{2}}{4 m}\left(\frac{\bar{\nabla}^{2} P}{P}-\frac{1}{2} \frac{(\bar{\nabla} P)^{2}}{P^{2}}\right)=0
\end{gathered}
$$

donde la ecuación (9) es una ecuación de continuidad para $P$ y la nueva fuerza que actúa sobre las partículas, el llamado potencial cuántico, está dado por

$$
U=-\frac{\hbar^{2}}{4 m}\left(\frac{\bar{\nabla}^{2} P}{P}-\frac{1}{2} \frac{(\bar{\nabla} P)^{2}}{P^{2}}\right)
$$

De esta manera, considerando el potencial total como $V_{\text {Total }}=V+U$, la ecuación 11 se puede reescribir como

$$
\frac{\partial S}{\partial t}+\frac{(\bar{\nabla} S)^{2}}{2 m}+V_{\text {Total }}=0
$$

la cual resulta ser una ecuación análoga a la de Hamilton-Jacobi (ecuación (7)), de tal manera que el momento se puede seguir calculando en la forma de la ecuación (8) y por lo tanto se pueden obtener las trayectorias de las partículas a través de la ecuación 14. El problema que surge es que las condiciones iniciales de nuestro sistema cuántico son desconocidas, por ello Bohm apela a la visión estadística de la función de onda y obtiene dichas condiciones iniciales a partir de la densidad de probabilidad $P=|\varnothing|^{2}$, no sin señalar que esta particularidad no obedece a la naturaleza conceptual de esta nueva descripción mecano-cuántica, si no debido "a nuestra ignorancia de las condiciones iniciales de la partícula" (Bohm 1952a, p. 171).

\subsection{La visión Bohmiana de la molécula de hidrógeno}

Ahora vamos a analizar los resultados obtenidos cuando la mecánica Bohmiana es aplicada al estudio de sistemas químicos, en particular al estudio de la molécula de hidrógeno. Si bien la teoría ha sido ampliamente utilizada para la resolución de problemas dentro de la física, nos abocaremos a este ejemplo, en tanto que es de sumo interés para la filosofía de la química.

Comenzaremos por examinar el átomo de hidrógeno, $H$. Para este caso el estado $1 s$ del electrón tiene la forma,

$$
\emptyset=f(r) e^{-\frac{i E t}{\hbar}}
$$

donde $E$ es la energía. Así, la función fase es $S=E t$ y, de acuerdo a la ecuación (8), el momento es $p=\nabla S=0$, lo que indica que el electrón se encuentra en reposo. No obstante, su posición es arbitraria y no podemos conocerla con exactitud, dado que las condiciones iniciales son desconocidas y se definieron estadísticamente. Aún con ello, el resultado va de acuerdo a la física clásica, ya que al no moverse el electrón en torno al núcleo no pierde energía y no colapsa con él, un resultado predicho por el electromagnetismo. Lo que si podemos determinar es la distancia a la que se encuentra el electrón del núcleo. Tenemos que la energía viene dada por

$$
V+U=E
$$

donde vemos que el potencial cuántico debe ser de naturaleza opuesta a la energía potencial y por tanto la equilibra. Cuando la energía corresponde a la del estado fundamental, la distancia entre el núcleo y el electrón corresponde al radio de Bohr. En cuanto a los demás orbitales, la fase es diferente de cero, $S \neq 0$, y depende de un ángulo $\phi$. En este caso se obtiene

$$
r=r_{0}, \grave{\mathrm{e}}=\grave{\mathrm{e}}_{0}, \phi=\phi_{0}+\frac{m \hbar t}{m_{0} r_{0}^{2} \sin ^{2} \grave{\mathrm{e}}_{0}}
$$

donde $r$, è, $\phi$ son las coordenadas polares, $r_{0}, \grave{\mathrm{e}}_{0}, \phi_{0}$ son las coordenadas iniciales, $m$ es el número cuántico azimuthal y $m_{0}$ es la masa del electrón. La ecuación (17) se interpreta que el electrón órbita 
alrededor del núcleo con un radio constante $\left(r_{0} \sin \grave{e ̀}_{0}\right)$ y con una velocidad angular proporcional a $\frac{\hbar}{m_{0} r_{0}^{2} \sin ^{2} \grave{e}_{0}}$ (Holland 1993, p. 150). Sin embargo, la órbita que traza el electrón es diferente de la usual órbita de Bohr, ya que en este caso dicha órbita no se encuentra en el plano ecuatorial, aunque si es paralelo a éste. Además, la cuantización no corresponde a la órbita, si no a la velocidad de la partícula.

Dentro de este entorno procederemos a examinar la molécula de hidrógeno, $H_{2}$. Antes, recordemos que en el ámbito de la química se dice que dos átomos se encuentran enlazados cuando comparten electrones, en el caso particular de la molécula de hidrógeno se habla de un enlace covalente. El problema surge cuando la mecánica cuántica trata de dar cuenta de la noción de enlace químico.

Dados dos átomos de hidrógeno en el estado $1 s$, y alejados el uno del otro, como se mencionó antes, lo electrones en este estado se encuentran en reposo. Cuando se van acercando dichos átomos para formar la molécula, la ecuación de onda independiente del tiempo correspondiente es

$$
\left[-\left(\frac{\hbar^{2}}{2 m}\right)\left(\nabla_{1}^{2}+\nabla_{2}^{2}\right)-\left(\frac{\hbar^{2}}{2 M}\right)\left(\nabla_{A}^{2}+\nabla_{B}^{2}\right)+V\right] \varnothing=E \varnothing
$$

donde $\psi=\psi\left(\bar{x}_{1}, \bar{x}_{2}, \bar{r}_{A}, \bar{r}_{B}\right)$, con $\bar{x}_{i}(i=1,2)$, son las coordenadas de los electrones, $\bar{r}_{j}, j=A$, $B$, son las coordenadas de los núcleos, $m$ es la masa del electrón, $M$ es la masa del núcleo. Además la energía potencial está definida como

$$
V=-\frac{e^{2}}{r_{A 1}}-\frac{e^{2}}{r_{B 1}}-\frac{e^{2}}{r_{A 2}}-\frac{e^{2}}{r_{B 2}}+\frac{e^{2}}{r_{12}}+\frac{e^{2}}{r_{A B}}
$$

donde $r_{\text {áâ }}$ son las distancias entre las partículas. La ecuación (18) puede ser resuelta empleando los métodos convencionales, obteniéndose así dos soluciones posibles, correspondientes a la función de onda enlazante y antienlazante, y cuya energía asociada a cada caso está dada por

$$
E_{ \pm}=Q_{ \pm}+V
$$

De acuerdo a los cálculos se observa que el potencial cuántico es el responsable de la interacción de los electrones, que conduce finalmente a la formación del enlace químico. Adicionalmente, de la ecuación (20) se sigue que la fuerza neta que actúa sobre los electrones es cero, lo cual indica que dichos electrones permanecen en reposo, y considerando la densidad de probabilidad se infiere que la posición más favorecida para encontrar a los electrones, resulta ser en la región entre los núcleos. Este resultado es muy notable, ya que la mecánica Bohmiana parece recuperar la noción de enlace propuesta por Lewis.

\section{5. ¿Dónde queda la TCAEM?}

En los comienzos de la mecánica cuántica (MC), Schrödinger trató de asociarle un significado físico a la ecuación de onda, proponiendo que ésta representaba algún proceso de vibración en el átomo, (Schrödinger 1926a) y que dicha concepción era más verosímil que aquella en la que se hablaba de los orbitales que albergaban electrones. Sin embargo, más tarde, contradiciendo sus primeras ideas, él indicó claramente que la ecuación de onda no debía ser empleada directamente para la interpretación del fenómeno físico, sino únicamente para obtener la cantidad $\emptyset^{*} \varnothing$, con la cual él define la densidad de electricidad, contrariamente a Born, quien define la misma cantidad como una densidad de probabilidad, en la forma $|\varnothing|^{2}$. De acuerdo con Bader, la interpretación probabilística de la función de onda $\emptyset$, no es consistente con los experimentos de rayos $\mathrm{X}$ empleados para medir la densidad electrónica en cristales (Bader 2011a), un hecho empírico que refuerza la idea planteada por Schrödinger. Por otro lado, si bien la mecánica Bohmiana (MB) descarta esta idea de una probabilidad ontológica asociada a la función de onda, no deja de asociarle un significado físico, argumentando que se trata de la representación matemática de un campo objetivamente real (Bohm 1952a, p. 170), el cual ejerce 
una fuerza sobre el electrón, de manera similar a como lo hace el campo electromagnético sobre una carga eléctrica, y tal campo $\varnothing$ obedece la ecuación de Schrödinger. En este sentido vemos que la TCAEM parece plantear una nueva concepción respecto de la función de onda, restándole significado físico y asociándola a la cantidad $\emptyset^{*} \varnothing$, de la cual se deriva el concepto de densidad electrónica $\tilde{\mathrm{n}}(r)$, que sí tiene un significado físico ya que determina la forma de la materia en el espacio real (Bader 2011a).

Bajo este mismo tenor, el hecho de que la TCAEM no interprete la función de onda $\varnothing$, de la misma manera como lo hace la $\mathrm{MC}$, inmediatamente excluye varios de los problemas que presenta la segunda. Por ejemplo, la cuestión sobre el principio de superposición resulta inexistente en la TCAEM, ya que en ésta no se habla siquiera de estados, en cambio está fuertemente fundamentada en magnitudes observables. Y a este respecto, las propiedades de una molécula descritas por la TCAEM, deben ser mensurables. De ahí que en esta teoría, la densidad electrónica tenga asociado un operador al estilo de un observable de Dirac, siendo así la propiedad medible del sistema más importante, ya que codifica los conceptos relativos a la estructura molecular. Es en términos de la densidad electrónica que se define la condición de contorno de un sistema abierto (un átomo en una molécula), que es la superficie de flujo cero que limita a un átomo y asimismo la noción de vía de enlace, indicador de que dos átomos se encuentran enlazados. En este punto es que la MB puede parecer similar a la TCAEM, puesto que la primera habla sobre trayectorias de partículas que están bien definidas, y la segunda define estas propiedades, la superficie de flujo cero y la vía de enlace, en términos de las líneas del campo vector gradiente de la densidad electrónica. Adicionalmente, por medio de la TCAEM se pueden construir las gráficas moleculares de los sistemas químicos, y estas representaciones recuperan la clásica idea de estructura molecular en términos de "barras y esferas", donde se puede ver claramente que un par de átomos enlazados, están unidos por medio de una acumulación de densidad electrónica entre ellos, resultado similar al de la MB. No obstante esta similitud, las trayectorias de las partículas en la $\mathrm{MB}$ son prácticamente imposibles de observar, a pesar de que son consistentes con el resultado que se observa, mientras que las vías de enlace de la TCAEM son, en principio, cantidades observables.

El siguiente aspecto trata sobre la individualidad de los sistemas que se estudian con estas teorías. En la MC no existe un criterio de individualidad, debido principalmente al formalismo matemático en el que se encuentra fundamentada la teoría, que evita que un sistema cuántico quede definido completamente a través de sus propiedades. Además, cuando dos sistemas cuánticos interaccionan forman un nuevo sistema, en el que ya no se reconocen los sistemas de los que está compuesto y debe ser tratado de manera holista. Por otro lado, dentro de la MB, sí se puede hablar de la individualidad de las partículas, ya que éstas poseen trayectorias determinadas que las definen a lo largo del tiempo. En el caso de la MB, la justificación de las estadísticas de Fermi-Dirac para el caso de los fermiones y la de Bose-Einstein en el caso de los bosones no tiene sus raíces en la indistinguibilidad de las partículas, sino en las propiedades del campo $\emptyset$. Ahora, en lo que respecta a la TCAEM, vemos que una molécula es una colección de átomos, y que cada átomo que la compone contribuye a las propiedades de dicha molécula. La condición para que se cumpla esto es que se pueda particionar la energía del sistema entero, en contribuciones atómicas, lo cual conduce a definir la superficie de flujo cero que limita a cada átomo dentro de la molécula (Bader 2007). En este sentido, ésta condición de contorno nos asegura que la densidad electrónica asociada a cada uno de los átomos no cambie, si bien tampoco impide que dicho átomo intercambie materia y energía con su vecino. Dicho de otro modo, los átomos que conforman una molécula no pierden su individualidad, en tanto conservan su densidad electrónica inalterable en el tiempo y ésta es la que define su contribución a las propiedades de la molécula, y aún más, a las propiedades de cualquier molécula de la que formen parte.

Una cuestión más a considerar, es el hecho de que Bader plantea la posibilidad de emplear los teoremas de la mecánica cuántica en la interpretación del enlace químico (Bader 2011b), propuesta que ya antes había sido introducida por Slater en 1933. De esta manera el lenguaje empleado en la TCAEM hace uso del concepto de fuerza, como son la fuerza de Feynman y la fuerza de Ehrenfest, las cuales a través del teorema del Virial son relacionadas con la energía del sistema, y sus contribuciones cinética y potencial (Bader 2011b). Lo interesante es que este lenguaje resulta ser poco congruente 
dentro del contexto de la MC, en contraste con la MB, en donde parecería encajar mucho mejor, dado que se acerca a una concepción más clásica sobre los sistemas cuánticos.

Considerando lo anteriormente expuesto, vemos que la TCAEM guarda una más estrecha relación con la $\mathrm{MB}$ que con la MC, debido principalmente a su carácter más clásico y determinista, que se yuxtapone con la visión en la que está basada la MC.

\section{Conclusiones}

En el presente trabajo hemos abordado la cuestión de la reducción de la química molecular a la mecánica cuántica desde la perspectiva de la TCAEM. Esta teoría se fundamenta en el hecho de que la densidad electrónica contiene toda la información física de la materia y es a través de las características topológicas de su distribución que se pueden definir los conceptos relacionados a la estructura molecular, además da cuenta de la noción de enlace químico en términos de fuerzas empleando los teoremas de la mecánica cuántica. Hemos argumentado que esta visión se contrapone con aquella referente a la MC, debido principalmente a que la segunda le da cierto significado físico de estado a la función de onda y de ahí se sigue el desarrollo de su formalismo que en última instancia acarrea las dificultades mencionadas anteriormente. Adicionalmente, en la $\mathrm{MC}$ no existe un criterio de individualidad que se debe a su naturaleza indeterminista de partículas indistinguibles, mientras que en la TCAEM, los átomos sí poseen tal criterio, ya que su densidad electrónica no se ve alterada en el tiempo, y los define en cualquier molécula a la que pertenezcan. Un resultado muy importante, ya que permite que las propiedades de un átomo puedan ser transferidas a la molécula en la misma medida que lo es su densidad electrónica.

Por otra parte, hemos comparado la TCAEM con la MB, y si bien la segunda guarda un nexo muy importante con la MC, a causa de que también le asocia un significado físico a la función de onda, como un campo-ø, y sigue empleando la cantidad $|\varnothing|^{2}$ como una densidad de probabilidad, vemos que resultan teorías más afines en los presupuestos que plantean. Mientras la MB habla acerca de trayectorias de partículas y define el enlace químico como la unión de dos átomos a través de electrones localizados entre ellos. La TCAEM propone la existencia de vías de enlace, como zonas donde se acumula la densidad electrónica, y con ello recupera representaciones clásicas de moléculas. Además, el criterio de individualidad de los sistemas que estudian, es otro atributo que comparten, dado que en la MB las partículas siguen trayectorias que las definen. Con todo, a pesar de las similitudes que guardan estas teorías, no dejan de ser hasta cierto punto ajenas.

Podemos decir que la TCAEM comparte rasgos con la MC, pero sobre todo con la MB. No obstante es una nueva teoría que se ocupa en particular de dar cuenta de los sistemas químicos, en tanto las otras dos teorías resultan ser más generales en sus aplicaciones, una cualidad que las separa y lleva por caminos diferentes.

\section{Bibliografía}

Bader, R. (1991), "A Quantum Theory of Molecular Structure and Its Applications”, Chemical Review 91: 893-928.

Bader, R. (1994), Atoms in Molecules. A Quantum Theory, Oxford: Clarendon Press.

Bader, R. (2005), "The Quantum Mechanical Basis of Conceptual Chemistry”, Monatshefte für Chemie 136: 819-854.

Bader, R. (2007), "Everyman's Derivation of the Theory of Atoms in Molecules", Journal of Physical Chemistry A 111: 7966-7972.

Bader, R. (2009), “Bond Paths Are not Chemical Bonds”, Journal of Physical Chemistry A 113: 10391-10396.

Bader, R. (2010), "The Density in Density Functional Theory", Journal of Molecular Structure 943: 2-18.

Bader, R. (2011a), "On the Non-Existence of Parallel Universes in Chemistry", Foundations of Chemistry 13: 11-37. 
Bader, R. (2011b), "Worlds Apart in Chemistry: A Personal Tribute to J. C. Slater", Journal of Physical Chemistry A 115: $12667-12676$.

Ballentine, L. (1998), Quantum Mechanics: A Modern Approach, Singapore: World Scientific Publishing Company.

Bohm, D. (1952a), "A Suggested Interpretation of the Quantum Theory in Terms of Hidden Variables. I", Physical Review 85: 166-179.

Bohm, D. (1952b), "A Suggested Interpretation of the Quantum Theory in Terms of Hidden Variables. II", Physical Review 85: 180-193.

Bricmont, J. (2016), Making Sense of Quantum Mechanics, Switzerland: Springer.

de Broglie, L. (1924), “A Tentative Theory of Light Quanta”, Philosophical Magazine Letters 47: 446-458.

Bub, J. (1997), Interpreting the Quantum World, Cambridge: University Press.

French, S. y D. Krause (2006), Identity in Physics: A Historical, Philosophical and Formal Analysis, Oxford: Oxford University Press.

Goldstein, H., Poole, C. P. y J. L. Safko (2002), Classical Mechanics, 3rd ed., San Francisco: Addison Wesley.

Heitler, W. y F. London (1927), "Interaction Between Neutral Atoms and Homopolar Binding According to Quantum Mechanics", Zeitschrift für Physik 44: 455-472.

Holland, P. (1993), Quantum Theory of Motion, Cambridge: Cambridge University Press.

Landau, L. y E. M. Lifshitz (1972), Mecánica cuántica no-relativista, Barcelona: Reverté.

Landau, L. D. y E. M. Lifshitz (1975), Mechanics, Amsterdam: Elsevier.

Matta, C. (2013), "Philosophical Aspects and Implications of the Quantum Theory of Atoms in Molecules (QTAIM)", Foundations of Chemistry 15: 245-251.

Matta, C. F., Massa, L. y T. A. Keith (2011), "Richard F. W. Bader: A True Pioneer", Journal of Physical Chemistry A 115: $12427-12431$.

Schrödinger, E. (1926a), "Quantisierung als Eigenwertproblem (Erste Mitteilung)", Annalen der Physik 79: 361-376. (Versión inglesa: "Quantisation as a Problem of Proper Values. Part I", en Schrödinger, E., Collected Papers on Wave Mechanics, London/Glasgow: Blackie \& Son Limited, 1928, pp. 1-12.)

Schrödinger, E. (1926b), “Quantisierung als Eigenwertproblem (Vierte Mitteilung)”, Annalen der Physik 81: 437-490. (Versión inglesa: "Quantisation as a Problem of Proper Values. Part IV", en Schrödinger, E., Collected Papers on Wave Mechanics, London/Glasgow: Blackie \& Son Limited, 1928, pp. 102-123.)

Schrödinger, E. (1926c), "An Undulatory Theory of the Mechanics of Atoms and Molecules", Physical Review 28: 1049. 1070.

Slater, J. C. (1933), "The Virial and Molecular Structure”, Journal of Chemical Physics 1: 687-691.

Tumulka, R. (2004), "Understanding Bohmian Mechanics: A Dialogue”, American Journal of Physics 72: 1220-1226. 